\title{
El deber procreativo de Jonas y el problema de la no-identidad
}

\author{
Jonas' procreative duty and the problem of non-identity
}

DANIEL LOEWE $@$ ia

\section{Resumen}

En su ética de la responsabilidad argumenta Jonas a favor de un imperativo categórico, pero heterónomo: que la humanidad sea. Según este, habría un deber procreativo de la humanidad. Este deber ya incluiría las condiciones de una vida genuinamente humana. En primer lugar, el artículo sostiene que este imperativo no es capaz de lidiar satisfactoriamente con el desafío impuesto por el problema de la no-identidad, popularizado de Parfit. Si la elección entre dos políticas hoy, implica la no existencia de generaciones en el futuro, o una existencia en malas condiciones o incluso en condiciones de un infierno terrenal, el imperativo de Jonas debiese llevar a escoger la segunda política. Esto es contraintuitivo. En segundo lugar, el artículo sostiene que la integración de los intereses de las generaciones futuras en la ética sí se puede realizar desde una interpretación kantiana. Y finalmente el artículo sostiene que el cumplimiento del deber de que la humanidad sea, puede llevar a violar la autonomía de las personas en el presente en pos de la generación de valor en el futuro.

Palabras clave: Ética de la responsabilidad. Deber procreativo. Kant. Problema de la noidentidad. Parfit.

\begin{abstract}
In his ethics of responsibility, Jonas argues for a categorical but heteronomous imperative: that humanity should be. According to this, there would be a procreative duty of humanity. This duty would already include the conditions of a genuinely human life. First, the article argues that this imperative is not capable of satisfactorily dealing with the challenge imposed by the problem of non-identity, popularized by Parfit. If the choice between two policies today implies the non-existence of generations in the future, or an existence in bad conditions or even in conditions of an earthly hell, Jonas' imperative should lead to the choice of the second policy. This is counter-intuitive. Secondly, the article argues that the integration of the interests of future generations into ethics can be done from a Kantian interpretation. Finally,

a Escuela de Gobierno, Universidad Adolfo Ibáñez, Santiago, Chile. Doctor en Filosofía, e-mail: daniel.loewe@uai.cl
\end{abstract}


the article holds that the fulfillment of the duty of humanity to be, can lead to violating the autonomy of people in the present in favor of the generation of value in the future.

Keywords: Ethics of responsibility. Procreative duty. Kant. The problem of non-identity. Parfit.

\section{Introducción'}

Como es conocido, Hans Jonas sostiene que los cambios y desafíos tecnológicos implican un cambio en la naturaleza de las obligaciones morales. El poder tecnológico y el crecimiento que conlleva ha llevado a que las éticas tradicionales no consideren el futuro como un problema, dado que lo consideran como dado e incluso como mejor que el presente. Sin embargo, nuestro enorme poder tecnológico pone en peligro, como nunca antes, la vida sobre la tierra. Así, el futuro como un espacio de desarrollo de la vida humana no está ya dado, y por tanto esta nueva ética debe hacerse cargo de aquel. La "ética de los vecinos" no sería capaz de dar una respuesta. Como es conocido, Jonas se hace cargo de esta nueva dimensión requerida con su "ética de la responsabilidad". Para esto, articula una reformulación del imperativo categórico kantiano. De modo más exacto, presenta cuatro variaciones -no completamente coincidentes- de esta reformulación. Las dos formulaciones positivas (la primera y la cuarta) son: "actúa de tal modo que las consecuencias de tu acción sean compatibles con la permanencia de genuina vida humana sobre la tierra", e "integra en tu elección actual la integridad futura de los hombres como objeto de tu voluntad" (1984, p. 36). Asegurar la posibilidad de "vida humana genuina" implica proteger el futuro de la autonomía, dignidad, integridad y vulnerabilidad de la humanidad.

De modo correcto Jonas sostiene que la falta de reciprocidad es problemática para muchas arquitectónicas éticas tradicionales (1984, p. 84). Y esta falta de reciprocidad se da entre los seres del presente y los del futuro (para una discusión, compare LOEWE, 2010; 2015). Sin embargo, hay articulaciones de la ética tradicional que sí permiten hacerse cargo de estos nuevos desafíos. El utilitarismo es probablemente la doctrina tradicional que mejor puede acometer este desafío -aunque es una doctrina en muchos sentidos criticable. E incluso, y contrariamente a lo que Jonas en ocasiones

\footnotetext{
${ }^{1}$ Agradezco a Juan Alberto Lecaros por comentarios de una versión previa de este artículo.

${ }^{2}$ Todas las citas de Hans Jonas son traducciones propias.
} 
sugiere, hay articulaciones de la ética de los derechos y los deberes que sí permiten hacerse cargo de los seres del futuro y sus intereses y necesidades. Incluso, como veremos, desde una perspectiva kantiana esto es posible. Pero Jonas sí tiene razón en que desde la arquitectónica kantiana no se puede obtener un deber para que haya vida humana en el futuro. Si bien de la expectativa de que pueda haber vida humana en el futuro se siguen las obligaciones hacia los seres humanos del futuro, de ella no se sigue que deba haber vida humana en el futuro.

Es contra esta posibilidad que la nueva ética de la responsabilidad de Jonas se tiene que posicionar. Es un posicionamiento ético exigente. En su interpretación no se trata sólo de una reformulación del imperativo categórico kantiano, de modo que las acciones de los seres humanos del presente sean compatibles con la posibilidad de la existencia de un futuro humano -y no humano (una posibilidad que se va actualizando sucesivamente con la reproducción, de modo que esta reformulación es compatible con la moral kantiana al ir generando deberes en el presente como una cadena que anticipatoriamente se extiende en el futuro), sino que la existencia humana debe realizarse en el futuro. Con sus propias palabras, el primer imperativo es que "el ser humano sea" (1984, p. 90). Recurriendo nuevamente a sus palabras: "un deber para la existencia de la humanidad futura" (1984, p. 86 y siguientes). Tomado en serio, este deber de existencia implica un "deber... reproductivo" (1984, p. 86), "aunque no necesariamente de cada individuo" (1984, p. 86). Se trata, en definitiva, de un deber procreativo de la especie humana.

En mi opinión esta es una pretensión inadecuada. Por una parte, supera con mucho lo que puede ser éticamente exigible si se respeta la autonomía individual (se trata, en definitiva, de una exigencia supererogativa que no debiese llegar a constituir un deber jurídico). Por otra parte, es insostenible una vez que se la despoja de todos sus supuestos metafísicos. No examinaré estos supuestos (en mi opinión, el realismo moral es insostenible $-\mathrm{y}$ la posición de Jonas articula un tipo de realismo moral), sino que asumiré que, mientras más exigentes los supuestos, menos probable es su aceptación y por tanto más improbable su influencia a nivel de leyes y políticas públicas. En este artículo argumentaré, primero, que del deber procreativo se siguen consecuencias poco convincentes al contrastarlo con el problema de la no-identidad. En segundo lugar, argumentaré que este deber implica una intromisión inaceptable en la autonomía, pero 
que, afortunadamente, este deber es innecesario para una ética de responsabilidad hacia el futuro. Finalmente obtendré algunas conclusiones.

\section{El deber procreativo y la no-identidad}

Jonas se pregunta si del hecho de que habrá personas en el futuro se sigue que tenemos la obligación de asegurarles ciertas condiciones de vida. O expresado de modo negativo, si el no cumplimiento de esta exigencia justifica una reclamación de parte de las generaciones futuras:

Porque de todos modos habrá seres humanos posteriores, cuando el tiempo llegue, su Existencia (Dasein) no pedida les otorga el derecho de reclamarnos (verklagen) a nosotros antecesores por ser autores de su infelicidad, si es el caso que mediante un actuar descuidado y evitable nosotros estropeamos el mundo o la constitución humana para ellos. Mientras que ellos sólo pueden hacer responsables de su existencia a sus procreadores directos [...], pueden hacer responsables por las condiciones de su existencia a los antepasados o en general a los autores de esas condiciones (1984, p. 88).

Según Jonas, si bien esto es correcto, no basta para la teoría ética. Y por dos razones (1984, pp. 88-89). En primer lugar, el pesimista podría hacer depender el imperativo o deseabilidad de la humanidad futura de las condiciones que se anticipan de su existencia, de modo tal que si los pronósticos son miserables no se los debiese traer a la existencia. En segundo lugar, la reclamación anticipada de las generaciones futuras no se sostendría si estas estuvieran de acuerdo con su suerte o incluso se sintieran bien con ella. Por eso no basta con dar por supuesta la existencia de generaciones futuras que tendrían una reclamación hacia nosotros por las condiciones de su existencia, como se sostiene en la cita. Inversamente podemos decir que la teoría ética requerida debe establecer normativamente que haya bumanidad con independencia de los pronósticos acerca de su calidad y tipo de vida, e independientemente del interés empírico de las generaciones futuras acerca de sus condiciones de vida. ${ }^{3}$

\footnotetext{
${ }^{3}$ Es corriente articular un argumento adicional de carácter trascendental con respecto al primer imperativo categórico de Jonas (por referencia a la idea de lo bueno, en 1984 pp. 155 y siguientes) de que la humanidad sea (por ejemplo, HÖSLE, 1994; APEL, 1992, p. 240). Según esta interpretación, la obligación procreativa de Jonas se daría porque su negación implicaría una contradicción performativa: al negar esta obligación doy cuenta de un propósito que la no realización de esta obligación haría imposible. Con otras palabras, dado que la condición para negar la obligación procreativa es la existencia, pero su negación implica la no existencia, su negación negaría la
} 
Se trata de un requerimiento muy exigente. $\mathrm{Y}$ este requerimiento es el que corporiza su primer imperativo: que debe haber humanidad (1984, p. 91) ${ }^{4}$. Según Jonas, la fundamentación de este imperativo es de tipo metafísico. Este imperativo nos hace responsable por la idea de humanidad, y la naturaleza de esta idea es tal que exige su presencia física en el mundo. Si bien en este texto no discutiré esta fundamentación, algunas notas esquemáticas son necesarias para situar la discusión que sigue.

Los pasos argumentativos son los siguientes (estos pasos están bien explicados en BERDINESEN, 2017). Para contestar a la pregunta última: “¿debe haber hombres en el futuro?" hay que enfrentarse a la pregunta metafísica fundamental: “¿Por qué debe haber algo en vez de nada?". Ahora bien, la respuesta a esta pregunta no es causal, sino que en el plano de la ética y del "deber" hay que considerar la pregunta acerca del valor: ¿qué significa valor? La idea de Jonas es que de la objetividad del valor se puede derivar un deber ser objetivo que nos hace responsable hacia el ser y su existencia. Y ese valor está inscrito ya en el organismo con sus propósitos: hay una autoafirmación del ser en toda vida biológica. Así, del ser del organismo (que no es sólo humano), se derivaría un deber ser (compare TRNKA, 2015). Ciertamente, toda esta estructura está muy lejos de los entendimientos actuales dominantes sobre las arquitecturas de la ética y la moral, en los que el hiato entre ser y deber ser es infranqueable - una brecha que Jonas, como es sabido, rechaza.

condición de existencia que hace posible negarla. Pero esto es un error. Por una parte, una contradicción performativa se da cuando el contenido proposicional de una declaración contradice las condiciones necesarias para su enunciación. El caso clásico es la afirmación "estoy muerto". Aquí, el contenido proposicional está en contradicción con las condiciones que permiten enunciarla. ¿Cuál es la contradicción entre el contenido proposicional de negar la obligación de procrear y las condiciones que hacen posible la enunciación de esta proposición? El error del argumento es considerar que la negación del deber de procreación es el deber de no procrear. Sólo en ese caso surgiría una contradicción performativa. Pero esto es un error: la negación de un deber procreativo no es la afirmación de un deber de no procrear, sino que la no obligación de procrear, que por cierto incluye el permiso de hacerlo. Por otra parte, como ha argumentado Angelika Krebs, la interpretación según la cual es una contradicción objetar la demanda que la humanidad debe ser preservada en razón de sus capacidades teleológicas superiores, porque al objetar se actúa teleológicamente y así se muestra que se aprecia esa teleología, se basa en una equivocación con respecto al tipo de valoración: valorar simultáneamente la teleología y objetar el valor que la humanidad tiene en razón de esta teleología, confunde el "valorar para uno mismo" con el "valor absoluto". El decidir dejar de procrear, de modo que en el futuro no haya sujetos que objeten el valor de la teleología, es compatible con valorar la teleología para uno mismo (1999, p. 127). Este argumento me parece inobjetable.

4 "Dass eine Menschheit sei", en ocasiones, Jonas lo formula como: "dass der mensch sein". Dado que Mensch se utiliza como un singular genérico, la traducción como "humanidad" es correcta. 
Si bien considero que esta estructura teoría no se sostiene, no argumentaré al respecto. Para mis propósitos lo interesante es que esta estructura se relaciona con problemáticas ontológicas. Como aquellas relativas a si agentes morales pueden tener deberes hacia seres no existentes; o si personas futuras (que llegarán a existir o que podrían llegar a existir) pueden tener derechos que impliquen obligaciones hacia los agentes morales del presente; o aquellas relativas al tipo de obligaciones que se puede tener hacia esos seres. Y esto nos sitúa en el centro del problema de la no-identidad popularizado por Parfit (1984; compare también 1976; 1983 -la discusión más completa de este problema en BOONIN, 2014; compare también LOEWE, 2019) -un problema que Jonas sólo puede sortear con un alto costo que torna implausible su modelo.

El problema de la no-identidad se funda en una paradoja en relación a los individuos futuros. A diferencia de la afectación en coetáneos, la afectación de nuestras acciones en seres del futuro no sólo refiere a la calidad de su existencia, sino también a su identidad y cantidad -es decir, quién llegará a existir y cuántos llegarán a existir. Este problema surge del reconocimiento de la contingencia de las condiciones de surgimiento de la existencia. Bajo el reconocimiento de la necesidad genética, según la cual cada ser particular sólo puede surgir de la confluencia de un cierto gameto femenino y otro masculino, y que esta confluencia está sujeta a condiciones temporales, se sigue que cada ser que llega a existir no podría haber existido si las condiciones de gestación (los gametos involucrados, o el timing) hubiesen sido otras. Bajo el supuesto de que la vida de todo aquel que llega a existir tiene un cierto valor mínimo, es decir, que se prefiera vivir a no haber existido, cada sujeto que llega a existir no puede reclamar que se lo ha dañado, aunque sus condiciones de vida sean pésimas, si evitar ese daño implica otras condiciones de gestación -porque en ese caso no hubiese llegado a existir, y para el sujeto es preferible vivir una vida que es valiosa de ser vivida, aunque sea en malas condiciones, que no haber llegado a existir.

Este problema teórico tiene consecuencias profundamente contraintuitivas en el caso de la ética o justicia intergeneracional. Al pensar en nuestra relación hacia los seres del futuro es corriente sostener que tenemos algún tipo de responsabilidad u obligaciones hacia ellos que van más allá de asegurar que su vida sea mínimamente valiosa de ser vivida. Así, tendemos a considerar que es moralmente incorrecto escoger cursos de acción que impliquen un nivel mínimo de calidad de vida para los seres del 
futuro, habiendo otros cursos de acción disponibles y con mejores resultados. Pero como estos cursos de acción (políticas energéticas, de consumo, etcétera) tienen consecuencias en quién procrea con quién y así, a lo largo del tiempo, en quién llegará a existir, en tanto los que lleguen a existir tengan una vida mínimamente valiosa no los podemos haber dañado y por tanto no tienen una reclamación moral hacia nosotros, aunque sus condiciones de vida sean pésimas. Hay múltiples versiones de este problema. De un modo esquemático están las variables individuales y las colectivas, de igual y de diferente número. En lo que sigue me concentraré en la colectiva de igual número, no sólo porque es relevante para los temas medioambientales, sino también porque es por relación a ella que se puede obtener un contraste claro con la propuesta de Jonas (aunque la versión colectiva de diferente número situaría a la teoría de Jonas bajo desafíos quizás aún mayores).

Imagine que una generación debe decidir entre dos opciones de política de desarrollo y explotación de recursos. La primera es una política de agotamiento de recursos. Si la escoge, los recursos serán utilizados de modo tal que durante los próximos doscientos años se elevará el nivel de vida de los que vivan. Sin embargo, luego de doscientos años se producirá una catástrofe medioambiental que implicará la muerte de muchos, y malas condiciones de vida para los sobrevivientes, así como su sufrimiento por la pérdida de familiares y amigos, etcétera -sin embargo, condiciones que aún estarán por sobre lo requerido para considerar que se trata de vidas valiosas de ser vividas.

La segunda es una política de explotación y uso moderado de recursos. Si escoge esta opción, el nivel de vida será ligeramente inferior al nivel que se sigue de la política de agotamiento de recursos por los próximos doscientos años. Pero luego de doscientos años no se producirá ninguna catástrofe medioambiental, de modo que el nivel de la calidad de vida se mantendrá y seguirá habiendo generaciones. En ambos casos la clase de individuos que llegará a existir será diferente.

La intuición compartida es que se debiese optar por la política de explotación y uso moderado de recursos, ya que, si se opta por la de agotamiento, se dañaría a los seres humanos del futuro. Y esta es también la intuición a la base de la reclamación de las generaciones futuras a la que Jonas refiere en el párrafo citado al comienzo de esta sección y que él considera correcta. Sin embargo, el problema de la no-identidad indica 
que esta intuición es normativamente incorrecta: no habría nada moralmente incorrecto en escoger la política depredadora de recursos, ya que los que lleguen a existir en malas condiciones de vida no habrían llegado a existir si se hubiese escogido la política de uso moderado, y dado que su existencia es aun valiosa de ser vivida, la elección de la política de agotamiento de recursos naturales no pudo haberlos dañado (en realidad jlos benefició con la existencia!).

Como veremos, la ética de la responsabilidad parece poder lidiar con el problema de la no-identidad. Sin embargo, si bien puede parcialmente respaldar algunas de nuestras intuiciones comunes al respecto, sus consecuencias son tales que resultan profundamente contraintuitivas al contrastarlas con otras de nuestras intuiciones. Quizás incluso más contraintuitivas que aceptar las consecuencias contraintuitivas del problema de la no-identidad.

La razón por la cual la ética de la responsabilidad parece respaldar una solución al problema de la no-identidad, se encuentra en el deber de que la humanidad sea -el primer imperativo de la ética de Jonas. Por sí mismo, este imperativo nos da una razón para rechazar la elección de la política de agotamiento de recursos naturales. Si se optase por esta política, por una parte, no habría humanidad después de doscientos años, lo que contradeciría el imperativo. Por otra parte, dado que su reformulación del imperativo categórico refiere a la compatibilidad de nuestras acciones con una vida "genuinamente humana", se debiese rechazar esta política porque la calidad de vida de la generación que vive la catástrofe sería probablemente tal que no correspondería a una vida genuinamente humana.

Pero este modo de hacerse cargo del problema no carece de dificultades. Esto se debe a que la confluencia de existencia humana y vida genuinamente bumana, que Jonas supone, no está dada. Y dado que Jonas sostiene que su primer imperativo (que la humanidad sea) es categórico, el cumplimiento del deber procreativo relacionado con el deber de existencia no puede depender de consideraciones relativas a la calidad de vida. Y esto tiene, como veremos, consecuencias profundamente contraintuitivas.

Jonas insiste en la prioridad del deber de existencia (Dasein) por sobre el deber de asegurar un cierto tipo de existencia (1984, pp. 88-90). ${ }^{5}$ Como vimos, Jonas rechaza

\footnotetext{
${ }^{5}$ Esta es la "Priorität der Pflicht zur Dasein", por sobre una "Pflicht gegen das Sosein der Nachkommen" (1984, pp. 88-90).
} 
que el deseo o deber de que haya humanidad futura dependa de las condiciones de su existencia que podamos prever. Así, el pesimista que anticipa pronósticos miserables no puede negar, según Jonas, la obligación procreativa. Sería justamente al revés: las obligaciones hacia un cierto tipo de existencia de las generaciones futuras, es decir una cierta calidad de vida, se siguen de la obligación primaria, más fundamental, de que existan. El pesimista que avizora malas condiciones de vida para las generaciones futuras, no debe prejuzgar la capacidad de las generaciones futuras de cargar con su existencia (1984, p. 90), sino que debe cumplir con el deber de la existencia, y derivativamente, con aquellos deberes hacia la realización de ciertas condiciones de vida (el Sosein de las generaciones futuras). ¿Qué condiciones de vida son estas? Dado que se trata de vida "genuinamente humana" podemos suponer que son aquellas condiciones fácticas que hacen posible este tipo de vida, es decir, que consideran la autonomía, dignidad, integridad y vulnerabilidad de la humanidad. Así, Jonas rechaza que eventualmente las generaciones futuras pudieses estar de acuerdo e incluso sentirse bien con una vida degradada, porque: "Ese tipo de consentimiento y bienestar sería lo último que le desearíamos a una humanidad futura, si precisamente se obtuviese al precio de la dignidad y llamado de los hombres" (1984, p. 88). Con un lenguaje contemporáneo se puede decir que Jonas propone una teoría suficientarista definida en base a un umbral establecido por las condiciones que hacen posible una "vida humana genuina". Es decir, el deber existencial implica un deber de asegurar condiciones de vida que hagan posible una vida genuinamente humana.

Proponer la prioridad del deber de existencia por sobre un deber relativo a generar ciertos tipos de existencia en vez de la situación inversa (es decir, de hacer depender el deber procreativo de la calidad previsible de vida de las generaciones futuras), evita conclusiones tan contraintuitivas como proponer un deber de traer a la existencia a todas aquellas personas que prospectivamente serán felices porque serán felices (lo que soluciona un cuerno del famoso problema de la asimetría (para un examen de este problema y una estrategia de solución, compare LOEWE, 2017)). Sin embargo, tiene consecuencias contraintuitivas. Consideremos dos situaciones.

De acuerdo a la solución umbralista del problema de la no-identidad, se sostiene que dañamos a las generaciones futuras toda vez que con nuestras acciones las traemos a una existencia que esté por debajo de ese umbral. El “daño” se relaciona con un criterio 
objetivo (un umbral) y no con la evaluación subjetiva que los individuos realicen de sus estados. Pero ahora imagine la siguiente elección. Si se escoge una política de uso moderado de recursos naturales las generaciones futuras tendrán una vida "genuinamente humana", mientras que si se escoge una depredadora tendrán una vida por debajo de ese umbral, pero una vida que todavía tiene valor de ser vivida. Imagine que la generación actual escoge la política de depredación. En este caso las generaciones futuras tendrán una mala calidad de vida, pero todavía valiosa de ser vivida. Parecería que estas generaciones futuras tendrían, tal como sostiene Jonas, un reclamo moral justificado sobre la generación actual, ya que no cumplió con la garantía del umbral objetivo. El problema es que esta estrategia no puede solucionar el problema de la noidentidad: la opción alternativa a la elección de la política de uso moderado de recursos hubiese implicado que ellas no habrían llegado a existir -pero, aunque su vida está por debajo del umbral, todavía es valiosa, de modo que vivir es mejor a no haber existido. Por lo tanto, no se los pudo haber dañado con la elección de esta política. Coincidentemente con las críticas usuales a las teorías umbralistas (como la de Jonas) se puede afirmar que las generaciones futuras estarían felices (glad) de renunciar a sus derechos (a una cierta calidad de vida definida mediante un umbral), con tal de existir. De este modo, no haríamos nada moralmente incorrecto al escoger la política depredadora y traer a estas generaciones a la existencia. La de Jonas reproduce el mismo problema de todas las estrategias que recurren a una teoría umbralista para dar cuenta del problema de la no-identidad.

Se puede argüir a favor de su estrategia que, dado que se trata una teoría umbralista definida mediante deberes y no mediante derechos, si se escoge la política depredadora se viola un deber, lo que debe ser evitado con independencia de si las generaciones futuras que sufren las consecuencias de esta violación estarían de acuerdo con ello. Jonas parece articular un argumento de este tipo. Así sostiene que en relación a las condiciones de las generaciones futuras no debemos preguntar acerca de los "deseos", sino por su "deber", que no depende de los hombres: "Esto quiere decir, que nosotros finalmente no consultamos el deseo anticipado de los que vendrán [...], sino que su deber (Sollen), que no está hecho por nosotros y que está por sobre nosotros" (1984, p. 89). Con otras palabras: el deber de las generaciones presentes es cuidar que las generaciones futuras tengan la capacidad de cumplir su deber de ser verdaderos 
humanos. ${ }^{6}$ En un cierto sentido esto es correcto. Pero sigue siendo misterioso en qué sentido el no cumplir con este deber de cuidado hacia las generaciones futuras se puede entender como daño. Si, como afirma Parfit, daño lo es siempre para alguien -aquel que se ve afectado-, en este caso no habría daño, dado que la alternativa habría sido la no existencia. Un modo alternativo para dar cuenta de lo moralmente incorrecto de la acción consiste en afirmar que se relacionaría no con el daño causado (que no habría), sino que con la violación de un deber categórico que, como todo deber categórico, vale con independencia de sus consecuencias y efectos en los intereses sensibles de los individuos. Así entendido, las generaciones futuras debiesen considerar que la generación presente violó una obligación categórica al escoger la política depredadora, y que esto es moralmente incorrecto, siendo absolutamente irrelevante que el beneficio de su existencia se retrotraiga a aquella violación de un deber categórico. En un mundo de carácter noumenal este sería el caso. Pero es implausible en un mundo fenomenal como el nuestro. Es razonable suponer que, ante la eventualidad de no haber existido en razón del cumplimiento de un deber categórico, las generaciones futuras que aún tengan algún disfrute de su existencia, estarían de acuerdo en privilegiar sus intereses sensibles por sobre sus intereses transcendentales.

Pero cualquiera sea la evaluación del caso examinado, las consecuencias más contraintuitivas de la estrategia de Jonas se expresan en un segundo caso. Imagine que el pesimista del argumento de Jonas tiene razón, pero en una versión aún más extrema. La decisión sería entre, por una parte, una política de uso moderado que luego de 200 años producirá pésimas condiciones de vida a las generaciones que sigan, condiciones muy por debajo de una vida genuinamente humana e incluso muy por debajo de una vida vivible. En este escenario los seres humanos del futuro tendrían vidas de permanente dolor y sufrimientos sin momentos de felicidad o sosiego. Algo así como los escenarios más dramáticos en el Jardín de las Delicias del Bosco. Llamémoslo el escenario del infierno terrenal. $O$, por otra parte, una política depredadora del medioambiente que provocará el fin de la vida humana sobre la tierra en 200 años. En este caso, dado que en el primer escenario se trata de vidas con una calidad de vida tal que se preferiría no existir a haber existido, sería razonable escoger la política depredadora y evitar así el infierno terrenal. Así, se evitarían existencias

\footnotetext{
6 "Pflicht zu wirklichem Menschentum".
} 
profundamente miserables y sin otra esperanza de redención que la muerte. Sin embargo, la prioridad absoluta del deber de existencia propuesto por Jonas, es decir del deber procreativo, implicaría que debemos traer a estos sujetos a la existencia, aunque su vida sea completamente miserable y sin esperanza. Esto me parece ser normativamente inaceptable.

Ciertamente Jonas imagina que no se puede suponer un consentimiento para la no existencia o para una vida inhumana de parte de la humanidad del futuro (1984, p. 80 -como la que se supondría para el evitar el infierno terrenal. vuelvo a esto en la próxima sección). Pero esta suposición es insostenible. Contra Jonas me parece que es factible y razonable suponer un consentimiento para la no existencia de parte de las generaciones futuras en el caso del infierno terrenal. Jonas parece pensar que la razón última para negar la validez de la pretensión de no existencia de las generaciones futuras es el deber categórico de ser, es decir, un deber incondicional que debe valer en toda circunstancia sin admitir excepciones. ¿Pero por qué debe valer este deber de existencia de la humanidad de modo categórico? Contra Jonas, el deber procreativo no puede tener una prioridad absoluta (lexicográfica, en conceptos más precisos) por sobre consideraciones acerca de la calidad de vida y mantener simultáneamente algún tipo de plausibilidad intuitiva.

\section{Deber procreativo y autonomía}

La insistencia de Jonas en un deber procreativo tiene un cierto sentido. Los problemas serios con respecto a una ética que incluya el futuro se dan al considerar espacios temporales amplios. Así, desde una perspectiva ética tradicional no es problemático incluir obligaciones hacia descendientes directos. Un modo de hacerlo (hay otros) es mediante la citada "responsabilidad en razón de autoría". Pero sí es problemático incluir obligaciones hacia individuos separados temporalmente de nosotros. Los casos de fines programados evidencian la dificultad: imagine que en razón de las decisiones de producción y consumo en la actualidad se escoge una política energética que implicará una buena calidad de vida para las personas, pero la imposibilidad de la vida humana a partir de 200 años. ¿'Tenemos un deber de no escoger esa política energética? ¿Y si la tenemos, en qué se basa? 
Como es conocido, en razón de la ampliación del poder humano de acción y así de la creciente inseguridad de las consecuencias a largo plazo, Jonas exige "la creación anticipada de la representación de las consecuencias lejanas" como el "primer deber de la ética del futuro" (1984, p. 64). Esta creación anticipada se debe hacer de modo intencional. Dado que las evaluaciones de la tecnología pueden ser erróneas, la ética de la responsabilidad de Jonas implica el principio de decisión "in dubio pro malo". Es decir, en caso de alguna duda acerca de las consecuencias distantes, se debe dar preminencia a los peores pronósticos. Esta es la "Heurística del temor" (1984, p. 64). De este modo, ya la posibilidad -aunque sea mínima- de un evento catastrófico, entendido como un evento que implique la imposibilidad de la vida humana, debiese llevar a descartar ese curso de acción (sin necesidad de contrastarlo evaluativamente con los posibles beneficios multiplicados por la probabilidad de su ocurrencia). Así, no es necesario que la política energética del ejemplo tenga como consecuencia la imposibilidad de la vida humana luego de 200 años, sino que basta la mínima posibilidad de que la pueda tener, para deber evitarla.

Por cierto, esta es una consecuencia lógica si, como hace Jonas, se sostiene el deber de que "haya humanidad". Este deber -y el deber procreativo de la especie que conlleva- es el que en última instancia está a la base de descartar cualquier curso de acción en razón de la más mínima posibilidad de una consecuencia catastrófica en el futuro. Si se trata de un deber categórico, como ciertamente lo es, debe valer siempre, sin admitir excepciones. De lo que se sigue que se debe descartar cualquier probabilidad de que se dé un escenario en que este deber sea violado -tal como el deber de no mentir se debe cumplir siempre, sin hacer depender su complimiento de ningún cálculo de probabilidades.

Pero no es necesario optar por un deber tan curioso como el deber procreativo de la humanidad para sostener que, en caso de probabilidades -aunque sean mínimasde eventos catastróficos, debemos rechazar aquellos cursos de acción asociados con aquellos. Desde una perspectiva kantiana esto es en principio posible. Como vimos, la moral kantiana no implica un deber de que deba haber humanidad, sino que un deber hacia los hombres en la medida que los haya. Pero esto ya basta para poder desarrollar un argumento. 
El aparataje teórico de Kant no sólo implica reconocer la existencia de obligaciones hacia los seres humanos que coexisten (la ética de los vecinos en su formulación más extendida), sino también hacia los que existirán en el futuro. Estas obligaciones se siguen del proceso de universalización característico de su estructura argumentativa (para un desarrollo de este argumento, compare LOEWE, 2018). El futuro, como un espacio que habitarán sujetos de derecho, sí implica obligaciones en el presente. Expresado de otro modo, dado su carácter universalizador, el imperativo categórico -con sus deberes negativos y positivos-implica obligaciones hacia todos los hombres, independientemente de su posición geográfica y temporal. En Ideas para una historia Universal en Clave Cosmopolita sostiene Kant incluso que ese aspecto de nuestros deberes hacia los seres humanos del futuro corresponde a nuestra naturaleza. ${ }^{7}$

Con otras palabras, el deber es incondicional, mientras que la localización geográfica o temporal de la existencia humana es contingente. Por lo tanto, en un marco teórico kantiano sí se pueden articular argumentos convincentes para, por ejemplo, no destruir la capacidad vital del planeta de modo tal que no podamos cumplir nuestras obligaciones hacia los seres humanos que existirán en el futuro. Por lo mismo, los escenarios de fines programados no son problemáticos para esta teoría.

Imagine que, traslapándose en la línea temporal, A tiene un deber hacia B, quien a su vez tiene un deber hacia $C$, con quien su existencia se traslapa parcialmente, pero que la existencia de A y C no se traslapa. ¿Podría A optar por una política energética que, como la mencionada, pone en peligro la posibilidad de que llegue a existir un $\mathrm{C}$ en razón de un evento catastrófico asociado probabilísticamente con la elección de esa política? A primera vista parece que sí. Después de todo, primero, C no está entre los existentes. Y, segundo: si razonablemente se puede hablar de deberes, ellos lo son hacia todos aquellos que lleguen a existir, pero no frente a todos aquellos que no llegarán a existir. Y en este escenario la generación C no llegará a existir. Sin embargo, este análisis no se sostiene. Si la universalización kantiana del imperativo categórico es temporal como he sostenido (y no sólo espacial), es decir, si los deberes que de este se desprenden lo son hacia todos aquellos que lleguen a existir (o si se prefiere otra terminología: son

\footnotetext{
7 Immanuel Kant, Idee zu einer allgemeinen Geschichte in weltbürgerlicher Absicht, Akademie Ausgabe, VIII: 27.
} 
deberes atemporales en el sentido que son siempre válidos en el tiempo ${ }^{8}-\mathrm{y}$ ciertamente en el espacio), que A opte por una política que ponga en peligro la existencia de C sería insostenible -pero no en razón de algún deber procreativo de la humanidad. La razón es que el deber de A frente a B, de no tratarlo como mero medio sino simultáneamente como fin en sí, como establece la fórmula de la humanidad del imperativo categórico (IV: 428), implica respetar su autonomía, incluyendo su autonomía reproductiva; y lo mismo vale en la relación entre $\mathrm{B}$ y C. Si con su elección de una política energética A torna imposible el desarrollo de la vida de C, A está violando la dignidad de B, al impedirle a este último su autonomía reproductiva y el cumplimiento de sus deberes frente a $\mathrm{C}$, aunque $\mathrm{C}$ nunca llegue a existir.

Un análisis similar se puede hacer en relación no sólo a la autonomía reproductiva, sino que a las condiciones fácticas que hacen posible la autonomía condiciones entre las que se debe contar algún sustrato medioambiental. ${ }^{9}$ Si en razón de la elección de $\mathrm{A}$, las condiciones fácticas hacen imposible la autonomía de $\mathrm{C}, \mathrm{A}$ está violando la dignidad de $\mathrm{C}$, aunque sus existencias no se traslapen y aunque no los hayan traído directamente a la existencia.

Se puede retrucar que un deber de mantener las condiciones fácticas que hacen posible la autonomía de las generaciones que lleguen a existir implica demasiado poco. Después de todo, es sabido que la autonomía kantiana requiere de pocas precondiciones exteriores (falta de recursos materiales no es, por ejemplo, un impedimento de la autonomía: en sentido kantiano, es posible ser autónomo en la más abyecta pobreza (KANT 1963, p. 121)). Este análisis es correcto. Pero la crítica injustificada. Después de todo, no toda autonomía debe ser kantiana, y una teoría de inspiración kantiana no está obligada a seguir su noción de autonomía. Por otra parte, la misma dificultad aplica a Jonas. Como vimos, para Jonas asegurar "vida humana genuina" implica proteger el futuro de la autonomía, dignidad, integridad y vulnerabilidad de la humanidad. Podemos entender autonomía, dignidad, integridad y vulnerabilidad de modo más o menos

\footnotetext{
${ }^{8}$ Una interpretación kantiana del "estatus moral" como "atemporal", es decir, uno que, desde el momento en que se da la existencia (bajo supuestos contingentes) se extiende hacia todos los tiempos (pasado y futuro), ha sido recientemente elaborada por Korsg aard (2018, cap. 5).

${ }^{9}$ Por ejemplo, según Grünewald, la fundamentación kantiana de la protección medioambiental y animal se debe construir sobre su filosofía completa, en tanto se considera: "el medioambiente... como el campo de la libertad que hay que proteger" (1988, p. 104. Trad. del autor). También O'Neill argumenta en este mismo sentido que es contradictorio universalizar una máxima de destrucción del medioambiente (1996, p. 176).
} 
exigente. Si las entendemos de modo poco exigente, entonces esta ética exigiría muy poco. Pero lo podemos entender de un modo más exigente (por ejemplo, incluso optando por una función igualitaria, aunque sería, por cierto, poco convincente). Y entonces puede exigir mucho $-\mathrm{y}$ lo mismo se puede hacer por referencia al entendimiento de la autonomía y dignidad de inspiración kantiana.

Ciertamente, la ética de la responsabilidad de Jonas no es kantiana. Si bien sostiene un deber incondicional de que el "el hombre sea" (con sus palabras: un deber incondicional de la humanidad de existir (1984, p. 80), lo que implica un deber procreativo de la especie, ella no se basa en la autonomía, sino que, como él mismo anuncia, es heterónoma. Esto se debe a que como vimos, de acuerdo a su metafísica, el deber está dado por el ser de las cosas, y no por la autonomía. (Como vimos, según Jonas, junto al propósito del organismo entra el valor al mundo -porque alcanzarlo es un bien, y no lograrlo es un mal). Es decir, el deber se impone a la voluntad de los sujetos como una constricción heterónoma dada por su ser, y no es el resultado del correcto uso de la razón. El contraste con Kant resulta evidente al considerar la formulación de la humanidad del imperativo categórico (IV: 428). Según esta, no se debe tratar a los otros exclusivamente como medios, sino que siempre simultáneamente como fines en sí. A su base está el respeto por la autonomía. Si yo trato a otro ser humano exclusivamente como medio violo su dignidad porque no respeto su autonomía. Así, en principio, si haciendo uso de su autonomía todas las mujeres decidiesen, cada una individualmente, dejar de procrear, los hombres no las podrían obligar a hacerlo sin violar su dignidad. Si lo hacen, las estarían tratando exclusivamente como medio, no como fines en sí.

Cuando Jonas se pronuncia contra el derecho de la humanidad al suicidio ("No hay derecho de la humanidad al suicidio" (1984, p. 80)), se imagina que si, idealmente, el hombre de Estado recurre al consentimiento de los gobernados, no puede suponer un consentimiento para la no existencia o para una vida inhumana de parte de la humanidad del futuro (1984, p. 80). Pero esto es incorrecto. Como vimos en la sección anterior, la humanidad del futuro que vive bajo condiciones inhumanas (Entmenschtsein) no estaría de acuerdo con ese tipo de existencia y preferiría incluso no haber existido. Jonas se equivoca al sostener que la humanidad del futuro jamás daría su consentimiento para no existir. Si la decisión es entre no ser, y ser bajo condiciones inhumanas (es decir 
condiciones que hagan de la vida una que carece del valor mínimo para ser vivido -el escenario del infierno terrenal), es razonable "suponer" que preferirían la no existencia por sobre la existencia miserable.

Pero como sea, lo interesante es que para Jonas el modo de existencia (el Sosein) no puede cualificar el deber de existir, que es un deber incondicional. Así afirma que, aun cuando pudiéramos suponer que la humanidad del futuro consentiría en no existir (lo que él considera es una "suposición demente" (irrsinige Annabme) -en lo que, como vimos, se equivoca), habría que rechazar ese consentimiento, ya que existe un deber incondicional de la humanidad de existir. Se trata, por tanto, de un deber categórico heterónomo.

Esto lo podemos ver en el por él sostenido deber reproductivo de la humanidad. Si bien afirma que este deber reproductivo no es "necesariamente de cada individuo" (1984, p.86), como su ética de la responsabilidad tiene su campo de acción en el espacio de la política, este deber sí puede tener (y en ciertas circunstancias debe tener) consecuencias constrictoras de la autonomía individual. El uso de la expresión "no necesariamente" lo deja en claro: si bien no es necesario que sea un deber de los individuos, lo puede ser, y es posible imaginar circunstancias en que lo debe ser. Imagine que los últimos y muy pocos humanos sobre la tierra leyeron a Benatar (2006) y su argumento los convenció, de modo que se transforman en antinatalistas filosóficos. Por lo tanto, sostienen que la vida humana es siempre mala y que hay un deber fundado filosóficamente para no reproducir a la especie. En este caso, como exigencia moral el imperativo reproductivo implicaría un deber individual de procrear. Y como exigencia política -no olvidemos que las exigencias de la ética de la responsabilidad son políticasimplicaría que, si existe la posibilidad de constreñir la voluntad de los antinatalistas obligándolos a procrear, cuando esta es la única posibilidad de cumplir el imperativo de que "la humanidad sea", es lo que se debe hacer. Evidentemente, este imperativo implica una constricción de la autonomía individual. Recordemos que es un imperativo categórico (no hipotético) pero heterónomo (y no autónomo), ya que está dado por el ser de las cosas. Así, si quedara sólo una pareja procreativamente capaz sobre la tierra, el imperativo procreativo implica que debe procrear. Pero si uno de ellos no quiere, vale el imperativo procreativo y el otro lo debe poder obligar (por ejemplo, mediante violación, o escondiendo los anticonceptivos). Esto se opone a un imperativo basado 
en la autonomía, según el cual no habría procreación. Con el segundo se acaba el propósito (se acaban los seres humanos) y por tanto el valor humano. Con el primero se mantiene el valor porque se mantienen los propósitos humanos, pero se viola la autonomía. ¿Qué mundo es mejor? ¿Aquel en que la vida humana continúa a pesar de que se viola la autonomía humana? ¿O aquel en que se respeta la autonomía, pero la vida humana se acaba? Yo tiendo a preferir el segundo al primero (después de todo, en ese mundo, todos aquellos que existirían en el mundo alternativo, no existirán, y, por lo tanto, no se violaría ninguna obligación hacia ellos). Jonas, por el contrario, preferiría el primero al segundo.

\section{Conclusión}

Jonas sostiene que la presencia del hombre era un dato del que partía cualquier idea de deber en el comportamiento humano. Pero ahora esta presencia estaría amenazada, por lo que esta misma presencia debe ser objeto de un deber: "el deber de garantizar en el futuro la premisa primera de todo deber, esto es, justamente la existencia de candidatos a un universo moral en el mundo físico" (1984, p. 34). Y garantizar la existencia de hombres en el futuro "implica, entre otras cosas, conservar este mundo físico de tal modo que las condiciones para tal existencia permanezcan intactas, lo que significa protegerlo, en su vulnerabilidad, contra cualquier amenaza que ponga en peligro esas condiciones" (1984, p. 34).

En mi opinión el deber de conservar este mundo de modo que la vida (humana y no humana) en el futuro sea posible es razonable. Por lo demás, y como vimos, sostener este deber no está necesariamente en oposición a una consideración ética deontológica como la kantiana. Pero el deber primero de Jonas no es garantizar las condiciones medioambientales que hagan posible una vida humana (este es, en sentido estricto, un deber derivativo), sino que el deber de que haya vida bumana en el futuro. Como vimos, se trata de un deber de procreación. El problema es que sostener la preeminencia de este deber, como Jonas lo hace, implica exigencias procreativas que nunca debiesen poder llegar a ser consideradas como un deber -al menos en tanto consideremos que la autonomía es importante. 


\section{Bibliografía}

APEL, K-O. The ecological crisis as a Problem for Discourse Ethics. En: ÖFSTI, A. Ecology and Ethics. Trondheim: Papir Trykk, 1992. p. 219-260.

BENATAR, D. Better Never To Have Been: The Harm of Coming into Existence. Oxford: Oxford University Press, 2006.

BERDINESEN, H. On Hans Jonas' "the imperative of responsibility". Philosophia, v. 17, p. 1628, 2017.

BOONIN, D. The Non-Identity Problem \& the Ethics of Future People. Oxford: Oxford University Press, 2014.

GRÜNEWALD, B. Natur und Praktische Vernunft. En: INGESIEP, G. \& ALTNER, G. Mensch, Umwelt und Philosophie. Bonn: Interdisziplinäre Beiträge, 1988.

HÖSLE, V. Ontologie und Ethik bei Hans Jonas. En: BÖHLER, D. Ethik für die Zukunft: Im Diskurz, mit Hans Jonas. Munich: Beck, 1994. p. 103-125.

JONAS, H. Das Prinzip Verantwortung. Versuch einer ethik für die technologische Zivilization. Frankfurt am Main: Suhrkamp, 1984/1979.

KANT, I. Grundlegung zur Metaphisik der Sitten. Akademie Ausgabe, volumen IV. Parcialmente disponible en: https://korpora.zim.uni-duisburg-essen.de/Kant/verzeichnisse-gesamt.html

KANT, I. Idee zu einer allgemeinen Geschichte in weltbürgerlicher Absicht. Akademie Ausgabe, volumen VIII. Parcialmente disponible en: https://korpora.zim.uni-duisburgessen.de/Kant/verzeichnisse-gesamt.html

KANT, I. Lectures on Ethics. New York: Harper Torchbooks, 1963.

KORSGAARD, C. M. Fellow creatures. Our obligations to the other animals. Oxford: Oxford University Press, 2018.

KREBS, A. Ethics of Nature. New York/Berlin: Walter de Gruyter, 1999.

LOEWE, D. Obligaciones hacia las generaciones futuras: el caso contractual. Veritas, v. 55, n. 1, p. 21-66, 2010.

LOEWE, D. Justicia contractual y los seres del futuro. En: FIGUEROA, M. Liberalismo Político. Problemas y Desarrollos Contemporáneos. Santiago, Chile: Ril editores, 2015. pp. 205-244.

LOEWE, D. La tesis de la asimetría: actualismo y el problema de la no-identidad. En: TRUCCONE, S. Justicia Intergeneracional. Ensayos desde el Pensamiento de Lukas H. Meyer. Argentina: Universidad Nacional de Córdova, 2017.

LOEWE, D. Environmental intergenerational justice and the non-identity problem: a Kantian approach. Environmental Ethics, v. 41 (4), 333-345, 2019.

O'NEILL, O. Towards Justice and Virtue. Cambridge: Cambridge University Press, 1996.

PARFIT, D. On doing the best for our children. En: BAYLES, M.D. Ethics and Population. Cambridge, Mass.: Schenkman Publishing Company Inc., 1976. p. 100-115. 
PARFIT, D. Future Generations: further problems. Philosophy and Public Affairs, v. 11, p. 113172, 1983.

PARFIT, D. Reasons and Persons. Oxford: Clarendon Press, 1984.

TRNKA, J. Hans Jonas on teleology in biology. The relevance of existential philosophy for biological thinking. Revista Interdisciplinaria de Filosofía y Psicología, v. 10, n. 34, p. 41-49, 2015. 